\title{
ALTERAÇÕES RADIOGRÁFICAS DO TÁLUS NO PÉ TORTO CONGÊNITO APÓS LIBERAÇÃO CIRÚRGICA PELA TÉCNICA DE MCKAY
}

\author{
RADIOGRAPHIC CHANGES OF THE TALUS IN CONGENITAL \\ CLUBFOOT AFTER SURGICAL RELEASE USING THE MCKAY PROCEDURE
}

\begin{abstract}
José Antonio Pinto', Andréa Canizares Hernandes², Thais de Paula Buchaim², Francesco Camara Blumetti ${ }^{3}$, Carla Chertman ${ }^{4}$, Patrícia Corey Yamane ${ }^{3}$, Artur da Rocha Corrêa Fernandes ${ }^{5}$
\end{abstract}

\section{RESUMO}

Objetivo: Analisar as alterações morfológicas do tálus após o tratamento cirúrgico do pé torto congênito pela técnica de McKay. Métodos: Foram analisadas, retrospectivamente, radiografias em perfil com carga dos pés de 14 pacientes com pé torto congênito unilateral submetidos ao tratamento pela técnica de McKay por dupla incisão. Todos os pacientes foram operados pelo mesmo cirurgião, com média de 6,53 anos entre a cirurgia e a radiografia. Comparamos as características do tálus dos pés operados com os parâmetros radiográficos dos pés contralaterais. Avaliamos a presença de deformidade do dômus e da cabeça do tálus (avaliação da esfericidade), a altura e o comprimento do tálus, a presença e grau de subluxação do navicular, a alteração do ângulo de Gissane e o padrão do trabeculado ósseo. Resultados: Alterações da cabeça do tálus ocorreram em 92,8\% dos casos; do dômus, em 92,8\%; e do trabeculado, em 100\%. A relação entre o comprimento do tálus do pé operado sobre o contralateral variou de 0,61 a 0,88 (média de 0,$79 ; \mathrm{DP}=0,09$ ), e da altura de 0,57 a 0,98 (média de 0,$82 ; \mathrm{DP}=0,12$ ). $\mathrm{O}$ ângulo de Gissane aumentou em todos os pés operados, e todos apresentaram subluxação do navicular, com índice variando de 6,43 a 59,75\% (média de 26,34\%; DP = 16,66\%). Conclusões: Alterações talares ocorreram em 100\% dos pés tratados pela técnica de McKay. Estabelecer parâmetros radiográficos para descrever e quantificar essas deformidades mostrou-se viável através de técnicas simples e de fácil execução.

Descritores-Pé Torto; Tálus; Radiografia; Procedimentos Cirúrgicos Operatórios; Morfologia

\section{ABSTRACT}

Objective: To analyze the morphologic changes of the talus after surgical treatment of congenital clubfoot using the McKay procedure. Methods: We retrospectively analyzed lateral standing radiographs of the feet in 14 patients with unilateral clubfoot treated by the McKay procedure. All patients were operated on by the same surgeon, with an average of 6.53 years between surgery and the radiograph. We compared the characteristics of the talus between the operated foot and the contralateral foot. We evaluated the deformity of the domus and the head of the talus (sphericity evaluation), the talar length and height, the presence and percentage of navicular subluxation, changes in the Gissane angle, and the trabecular pattern of the bone. Results: Deformities of the head of the talus were observed in $92.8 \%$ of the patients; of the domus in $92.8 \%$; and of the trabecular pattern in $100 \%$. The ratio between the talar lengths of the operated foot and the contralateral foot ranged from 0.61 to 0.88 (Mean 0.79, $S D=0.09$ ), while the height ratio ranged from 0.57 to 0.98 (Mean 0.82, $S D=0.12$ ). The Gissane angle was increased in all of the operated feet, which also showed some degree of navicular subluxation, ranging from 6.43 to $59.75 \%$ (Mean $26.34 \%, S D=16.66 \%$ ). Conclusions: Abnormal talar findings were observed in 100\% of the feet treated using the McKay procedure. The establishment of radiographic parameters to describe and quantify these deformities was feasible through simple and easy techniques.

Keywords - Clubfoot; Talus; Radiography; Surgical Procedures, Operative; Morphology

1 - Professor Adjunto e Chefe da Disciplina de Ortopedia Pediátrica do Departamento de Ortopedia e Traumatologia da Universidade Federal de São Paulo, São Paulo, Brasil.

2 - Médica Residente do Departamento de Ortopedia e Traumatologia da Universidade Federal de São Paulo, São Paulo, Brasil.

3 - Médico Assistente da Disciplina de Ortopedia Pediátrica do Departamento de Ortopedia e Traumatologia da Universidade Federal de São Paulo, São Paulo, Brasil.

4 - Médica Especializanda do Setor de Cirurgia do Pé e Tornozelo da Disciplina de Ortopedia do Departamento de Ortopedia e Traumatologia da Universidade Federal de São Paulo, São Paulo, Brasil.

5 - Professor Adjunto e Vice-Chefe do Departamento de Diagnóstico por Imagem da Universidade Federal de São Paulo, São Paulo, Brasil.

Trabalho desenvolvido na Disciplina de Ortopedia Pediátrica do Departamento de Ortopedia e Traumatologia da Universidade Federal de São Paulo -

Escola Paulista de Medicina.

Correspondência: Thais de Paula Buchaim - Rua Borges Lagoa, 783 - 5º Andar - Vila Clementino - 04038-032 - São Paulo, SP. E-mail: thaispb70@yahoo.com.br 


\section{INTRODUÇÃO}

O pé torto congênito equino cavo varo, caracterizase por uma deformidade causada principalmente pelo desalinhamento e malformação dos ossos e articulações do retropé. Apresenta incidência populacional média de 1:1.000 nascido vivos ${ }^{(1)}$ e trata-se de uma das anomalias congênitas mais estudadas pela comunidade científica ortopédica.

Em uma busca conduzida na literatura internacional e nacional, encontramos aproximadamente 700 artigos publicados sobre o tema nos últimos 10 anos. Entretanto, quando abordamos especificamente as complicações que dizem respeito às deformidades radiográficas encontradas no tálus durante e após o tratamento, este número se reduziu significativamente, resumindo-se a simples citações ou parágrafos dentro de trabalhos com outros enfoques.

Sabemos que geralmente as deformidades do tálus resultam de graus variáveis de necrose, complicação que geralmente passa despercebida durante o tratamento do pé torto congênito. Sua incidência aumenta quando o tratamento é cirúrgico, principalmente quando a técnica utilizada necessita de ampla liberação de partes moles. Entretanto, também encontramos descrições das deformidades do tálus do tipo flap top, classicamente relacionadas com a necrose, em pacientes submetidos ao tratamento não cirúrgico. Esta deformidade provavelmente é causada pela dorsiflexão forçada do pé, na tentativa de reduzir o equinismo, gerando um mecanismo de alavanca que deforma o molde cartilaginoso flexível deste osso ${ }^{(2)}$.

A vascularização do tálus se dá exclusivamente através de vasos que penetram no osso através de inserções capsulares e ligamentares, o que o torna particularmente suscetível à necrose avascular. Seu suprimento arterial extraósseo provém basicamente de ramos das artérias tibial posterior, tibial anterior e fibular ${ }^{(3)}$. Estes vasos formam vários plexos anastomóticos ao redor do tálus. $\mathrm{O}$ ramo calcâneo da artéria tibial posterior forma uma rede com o ramo fibular que irriga a tuberosidade posterior do tálus. A maior artéria do canal do tarso, também proveniente da tibial posterior, forma um rico plexo vascular com a artéria do seio do tarso (que apresenta contribuições da artéria tibial anterior e fibular). Estas anastomoses ocorrem dentro do canal do tarso e irrigam a maior parte do corpo central e da região lateral do tálus. Um ramo deltoideo menor surge na origem da artéria do canal do tarso e corre entre as partes talotibial e talocalcaneana do ligamento deltoide para suprir a parte medial do corpo do tálus. A artéria tibial anterior emite ramos para a cabeça e colo do tálus, com contribuições da artéria fibular e do ramo deltoideo da artéria tibial posterior.

Conhecendo-se o aporte circulatório do tálus, podese concluir que as técnicas mais lesivas são aquelas que preconizam a liberação peritalar, principalmente com a secção do ligamento deltoide. A técnica de McKay/Simons consiste na liberação subtalar completa com secção opcional do ligamento interósseo talocalcaneano, alongamento dos tendões flexores, secção completa do ligamento deltoide superficial e parcial do profundo e secção dos ligamentos bifurcados, calcaneonavicular plantar e talonavicular dorsal. Este procedimento pode ser realizado utilizando-se a incisão do tipo Cincinatti clássica ou uma dupla via lateral e medial ${ }^{(4)}$. Nestas circunstâncias, o conhecimento anatômico preciso é necessário para que as chances de osteonecrose sejam minimizadas.

\section{OBJETIVO}

O objetivo de nosso trabalho foi analisar as alterações morfológicas do tálus decorrentes do tratamento cirúrgico do pé torto congênito por meio da liberação peritalar.

\section{MATERIAL E MÉTODO}

Foram analisados, retrospectivamente, os prontuários de 76 pacientes com pé torto congênito provenientes de um hospital da rede pública de São Paulo. De forma a padronizar nossa amostra, incluímos apenas pacientes com pé torto congênito unilateral submetidos ao tratamento cirúrgico pela técnica de McKay, por dupla incisão, no período de 1996 a 2008. Todas as cirurgias foram realizadas pelo mesmo médico ortopedista. Um total de 14 pacientes satisfizeram os critérios de inclusão e foram avaliados neste trabalho. Suas características gerais estão sumarizadas na Tabela 1.

Para a avaliação radiográfica, utilizamos os últimos exames disponíveis nos prontuários dos pacientes. Foram analisadas as incidências de perfil com carga dos dois pés. $\mathrm{O}$ tempo médio entre a cirurgia e a radiografia controle utilizada na pesquisa foi de 6,53 anos (de dois a 12 anos). As características morfológicas do tálus dos pés operados foram comparadas aos parâmetros radiográficos dos pés normais contralaterais, sendo avaliada a presença de deformidade do dômus e da cabeça do tálus, a altura e o comprimento do tálus, a presença e grau de subluxação do navicular, a alteração do ângulo de Gissane e o padrão do trabeculado ósseo. 
Tabela 1 - Relação de pacientes envolvendo idade, sexo, lado acometido, data da cirurgia e data da radiografia avaliada.

\begin{tabular}{c|c|c|c|c|c}
\hline Paciente & Idade & Sexo & Lado & Data Cirurgia & Data Radiografia \\
\hline 1 & 11 & M & E & 1990 & $12 / 12 / 2006$ \\
\hline 2 & 7 & M & E & 2003 & $1996 / 11 / 2007$ \\
\hline 3 & 13 & M & D & 2002 & $10 / 04 / 2008$ \\
\hline 4 & 8 & M & E & 2002 & $05 / 03 / 2008$ \\
\hline 5 & 7 & M & E & 2003 & $09 / 11 / 2005$ \\
\hline 6 & 7 & F & E & 2001 & $04 / 11 / 2005$ \\
\hline 7 & 10 & M & E & 2002 & $07 / 02 / 2007$ \\
\hline 8 & 11 & M & E & 1999 & $06 / 06 / 2008$ \\
\hline 9 & 11 & M & D & 1999 & $12 / 02 / 2009$ \\
\hline 10 & 8 & M & E & 2000 & $10 / 03 / 2006$ \\
\hline 11 & 11 & F & E & 1999 & $26 / 11 / 2008$ \\
\hline 12 & 8 & M & D & 2001 & $06 / 03 / 2008$ \\
\hline 13 & & M & E & 2002 & $18 / 09 / 2008$ \\
\hline 14 & & E & & $14 / 05 / 2007$ \\
\hline
\end{tabular}

Para analisar a deformidade no dômus do tálus, avaliamos a esfericidade da articulação tibiotalar, utilizando uma régua de círculos concêntricos de acordo com o método de Mose. Os pés estudados foram subdivididos em três grupos, sendo eles: (A) normal, permitindo boa mobilidade da articulação; (B) levemente achatado; e (C) grosseiramente alterado ou plano (Figura 1).

Para avaliação da deformidade da cabeça, os pés foram divididos em três grupos de acordo com a esfe- ricidade da articulação talonavicular: (A) convexa; (B) plana; e (C) côncava (Figura 2).

Não encontramos na literatura estudada nenhum parâmetro preestabelecido para avaliação da altura ou do comprimento do tálus. Após testarmos diversas técnicas para realização de tais medidas, com auxílio de um médico radiologista, encontramos maior facilidade na padronização e reprodutibilidade entre as diferentes radiografias com as seguintes técnicas:
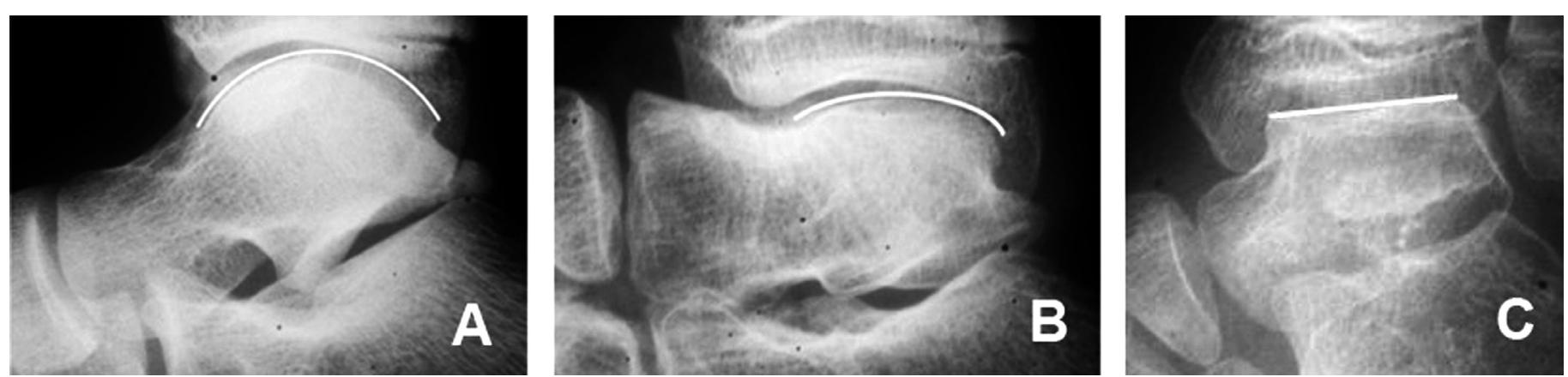

Figura 1 - Padrões de esfericidade do tálus: (A) normal, permitindo boa mobilidade da articulação; (B) levemente achatado; e (C) grosseiramente alterado ou plano.
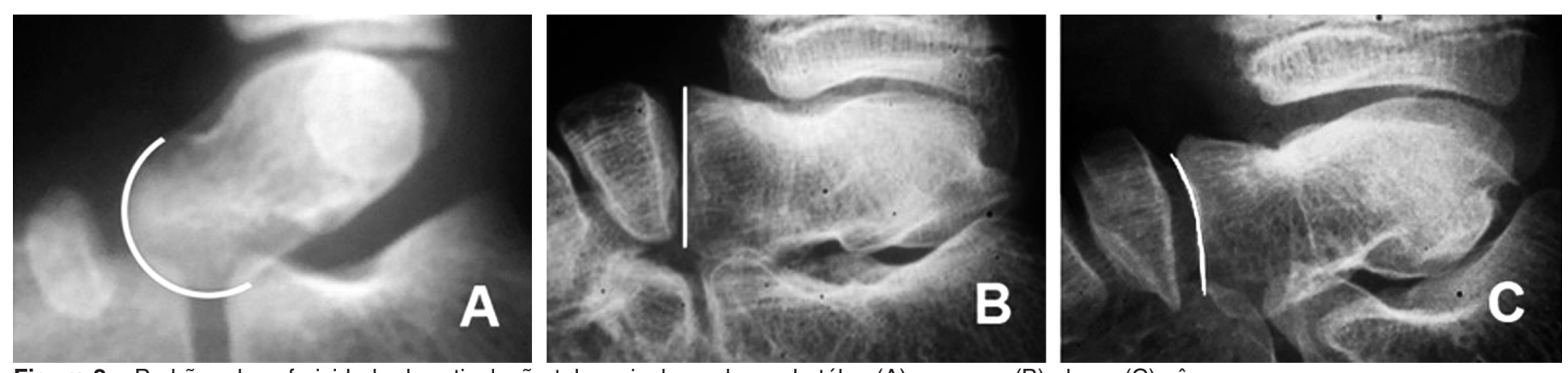

Figura 2 - Padrões de esfericidade da articulação talonavicular: cabeça do tálus (A) convexa; (B) plana; (C) côncava. 
Mensuração da altura (Figura 3):

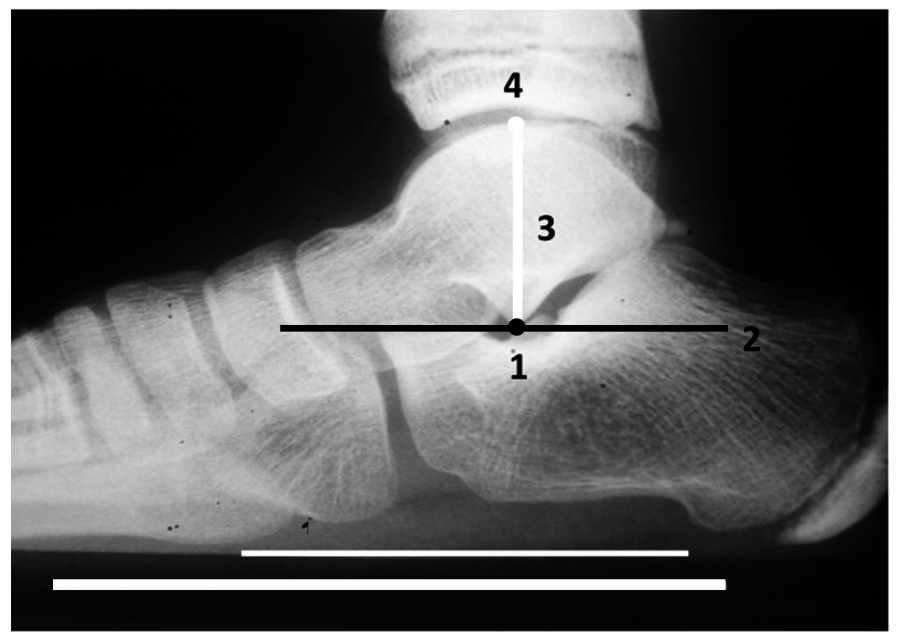

Figura 3 - Mensuração da altura talar.

1. Identificar o ápice do processo lateral do tálus (ponto 1);

2. Traçar reta paralela ao solo tangenciando este ponto (reta 2);

3. A partir do ponto 1 , traçar reta perpendicular à reta 2 (reta 3) até o ponto de intersecção com a superfície articular do dômus talar (ponto 4);

4. A altura do tálus é dada pelo comprimento da reta 2 do ponto 1 ao ponto

Mensuração do comprimento (Figura 4):

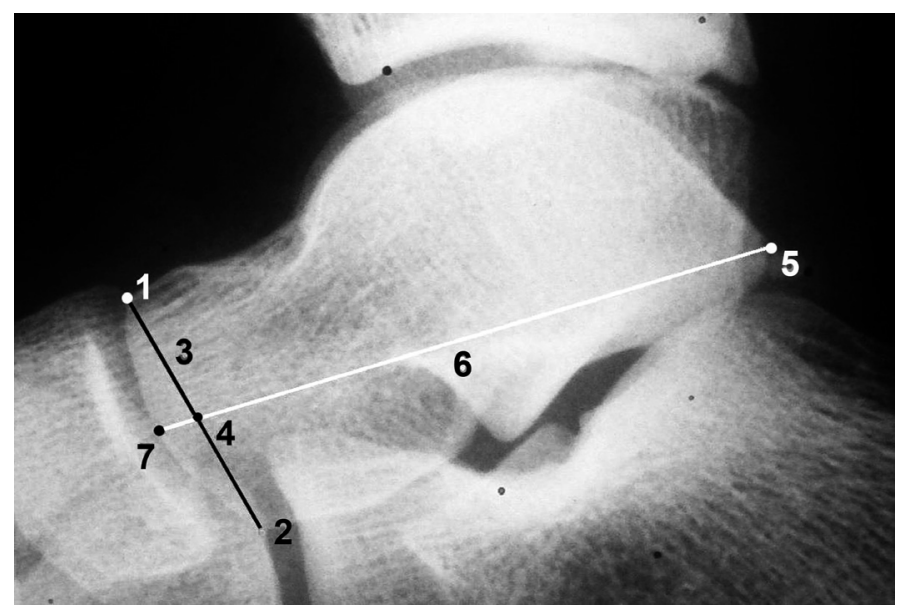

Figura 4 - Mensuração do comprimento talar.

1. Identificar o ponto mais superior (ponto 1) e o ponto mais inferior (ponto 2) da superfície articular do tálus da articulação talonavicular na radiografia em perfil;

2. Traçar reta ligando os pontos 1 e 2 (reta 3 );

3. Identificar o ponto médio da reta 3 (ponto 4);

3. Identificar o ponto mais posterior do processo posterior do tálus, não incluindo o os trigonum (ponto 5);

4. Traçar reta (reta 6) a partir do ponto 5, tangencian- do o ponto 4 até a intersecção com superfície articular do tálus na articulação talonavicular (ponto 7);

5. A distância do ponto 5 ao ponto 7 constitui o comprimento do tálus.

Foi realizada a mensuração da altura e do comprimento no pé acometido e no contralateral. A relação simples percentual foi então obtida entre tais medidas.

A alteração morfológica do tálus na articulação subtalar foi avaliada realizando-se a medida do ângulo de Gissane, que consiste no ângulo entre a reta que tangencia a faceta articular anterior e a reta que tangencia a faceta articular posterior do tálus. $\mathrm{O}$ ângulo foi medido em ambos os pés de cada paciente e, a seguir, foi calculada a diferença entre o pé acometido e o pé normal.

Para análise do grau de subluxação do navicular na articulação talonavicular, estabelecemos um índice, calculado da seguinte maneira (Figura 5):

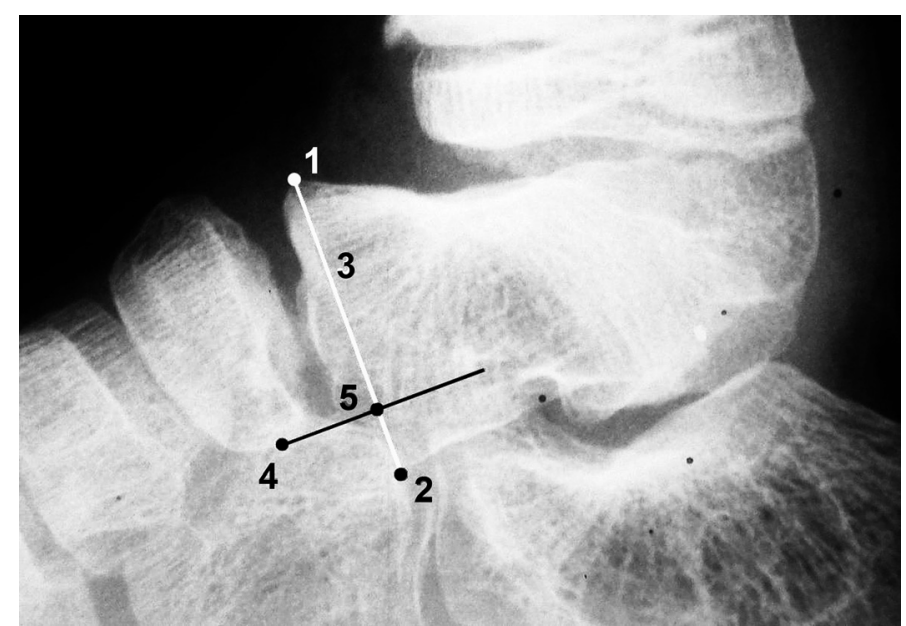

Figura 5 - Cálculo do grau de subluxação do navicular.

1. Identificar o ponto mais superior (ponto 1) e o ponto mais inferior (ponto 2) da superfície articular do tálus da articulação talonavicular na radiografia em perfil;

2. Traçar reta ligando os pontos 1 e 2 (reta 3);

3. Traçar reta perpendicular à reta 3 , tangenciando o ponto mais inferior do navicular (ponto 4);

4. No ponto de intersecção das retas, encontra-se o ponto 5;

5. O índice é calculado fazendo-se a relação da distância entre os pontos 2 e 5, sobre a distância entre os pontos 1 e 2.

Para avaliação do padrão do trabeculado ósseo do tálus dos pés operados em relação aos pés normais, subdividimos os casos em três grupos, de acordo com o sentido predominante das trabéculas ósseas nas radiografias estudadas: (A) horizontal: predominância de trabéculas horizontais; padrão compatível com tálus normal; (B) 
misto: presença de trabéculas verticais e horizontais, igualmente distribuídas; e (C) vertical: predominância de trabéculas orientadas verticalmente.

\section{RESULTADOS}

Os resultados da avaliação radiográfica estão ilustrados na Tabela 2.

Das 14 radiografias avaliadas, $85,71 \%$ (12 pacientes) apresentavam achatamento da cabeça do tálus operado em relação ao contralateral, $7,14 \%$ (um paciente) apresentavam convexidade e $7,14 \%$ (um paciente) apresentavam concavidade.

Quanto à deformidade do dômus talar do pé operado, encontramos $71,43 \%$ (10 pacientes) dos casos com forma elíptica, $21,43 \%$ (três pacientes) com forma plana e 7,14\% (um paciente) com forma esférica.

Todos os casos apresentaram redução de pelo menos $10 \%$ do comprimento, sendo que, destes, metade apresentou redução de pelo menos $20 \%$. A relação do comprimento do tálus do pé operado sobre o contralateral variou de 0,61 a 0,88, com média de 0,7929 ( $\mathrm{DP}=0,0905)$.

A altura do tálus dos pés operados foi menor do que a do contralateral em todos os casos avaliados, sendo que a relação variou de 0,57 a 0,98 , com média de 0,8171 $(\mathrm{DP}=0,11796)$, havendo uma redução de pelo menos $10 \%$ na maioria dos casos $(71,43 \%)$.

Nos pés operados, nenhum tálus apresentou padrão trabecular normal (predominantemente horizontal), sendo que $57,14 \%$ deles apresentaram padrão misto (vertical e horizontal) e 42,86\% apresentaram padrão predominantemente vertical.

O ângulo de Gissane apresentou-se aumentado em todos os pés operados, quando comparado ao contra- lateral. Esse aumento variou de 2 a $40^{\circ}$, com média de $20,64^{\circ}$, sendo que, em 10 casos $(71,43 \%)$, esse aumento foi de pelo menos $20 \%$.

Todos os casos apresentaram algum grau de subluxação do navicular na articulação talonavicular, sendo que seu índice variou de 6,43 a 59,75\%, com média de $26,34 \%(\mathrm{DP}=16,66 \%)$. Dos 14 pés operados avaliados, nove $(64,28 \%)$ apresentaram pelo menos $20 \%$ de ascensão do navicular.

\section{DISCUSSÃO}

A literatura descreve inúmeras complicações em cirurgias de liberação extensa de partes moles no tratamento do pé torto congênito. No entanto, não existem critérios radiográficos bem definidos para o diagnóstico da necrose e outras alterações do tálus nesta doença. Desta forma, estabelecemos nossos próprios parâmetros radiográficos, baseados nas características morfológicas atípicas encontradas.

Há relatos de alterações na forma do tálus após o tratamento do pé torto congênito, tanto com a técnica cirúrgica quanto com a não cirúrgica ${ }^{(5-7)}$. Em 1980, Ponseti et $a l^{(8)}$ relataram alterações do comprimento total e forma do dômus e cabeça do tálus em seu consagrado protocolo de tratamento com gessos seriados. Cummings et $a l^{(9)}$ relataram alterações do tipo bone-within-the-bone, achatamento do dômus e hipoplasia do colo e cabeça talar, após o tratamento cirúrgico pela técnica de McKay. Diversos outros autores também relataram um número variável de alterações talares com diferentes técnicas de tratamento.

Observamos uma incidência aumentada (100\%) de alterações radiográficas do tálus, quando comparada à incidência habitual previamente descrita $(27-74 \%)^{(7-10)}$.

Tabela 2 - Relação de pacientes envolvendo os resultados dos parâmetros radiográficos avaliados.

\begin{tabular}{|c|c|c|c|c|c|c|c|}
\hline Paciente & $\begin{array}{c}\text { Deformidade } \\
\text { Cabeça }\end{array}$ & $\begin{array}{l}\text { Deformidade } \\
\text { Dômus }\end{array}$ & Relação Altura & $\begin{array}{c}\text { Relação } \\
\text { Comprimento }\end{array}$ & Trabeculado & $\begin{array}{c}\text { Aumento } \\
\text { Gissane }\end{array}$ & $\begin{array}{c}\text { Índice Subluxação } \\
\text { Navicular }\end{array}$ \\
\hline 1 & Achatada & Plano & 0,86 & 0,79 & Misto & $40^{\circ}$ & $25,12 \%$ \\
\hline 2 & Achatada & Elíptico & 0,92 & 0,84 & Misto & $30^{\circ}$ & $41,17 \%$ \\
\hline 3 & Achatada & Elíptico & 0,57 & 0,84 & Vertical & $7^{\circ}$ & $8,02 \%$ \\
\hline 4 & Achatada & Plano & 0,95 & 0,77 & Vertical & $20^{\circ}$ & $22,29 \%$ \\
\hline 5 & Achatada & Esférico & 0,91 & 0,75 & Misto & $20^{\circ}$ & $20,91 \%$ \\
\hline 6 & Convexa & Elíptico & 0,98 & 0,87 & Misto & $24^{\circ}$ & $58,80 \%$ \\
\hline 7 & Côncava & Elíptico & 0,86 & 0,86 & Misto & $30^{\circ}$ & $12,33 \%$ \\
\hline 8 & Achatada & Elíptico & 0,82 & 0,88 & Vertical & $2^{\circ}$ & $33,74 \%$ \\
\hline 9 & Achatada & Elíptico & 0,87 & 0,61 & Vertical & $30^{\circ}$ & $17,33 \%$ \\
\hline 10 & Achatada & Elíptico & 0,76 & 0,74 & Vertical & $22^{\circ}$ & $6,43 \%$ \\
\hline 11 & Achatada & Elíptico & 0,75 & 0,88 & Misto & $20^{\circ}$ & $17,06 \%$ \\
\hline 12 & Achatada & Elíptico & 0,84 & 0,86 & Vertical & $8^{\circ}$ & $23,71 \%$ \\
\hline 13 & Achatada & Plano & 0,7 & 0,8 & Vertical & $26^{\circ}$ & $59,75 \%$ \\
\hline 14 & Achatada & Elíptico & 0,65 & 0,61 & Vertical & $10^{\circ}$ & $22,19 \%$ \\
\hline
\end{tabular}


Provavelmente, este fato é consequência da técnica empregada pelo cirurgião na época, que consistia na técnica de McKay com liberação peritalar extensa e importante lesão do suprimento arterial do tálus.

Devido à escassez de trabalhos descritivos anatômicos radiográficos da deformidade talar no pé torto congênito, propusemos alguns parâmetros para uma avaliação mais exata desta complicação. Estes parâmetros são a altura e comprimento do tálus, grau de subluxação talonavicular, ângulo de Gissane, características da deformidade da cabeça e do dômus do tálus e padrão do trabeculado ósseo. O ângulo de Gissane já é uma medida consagrada na Ortopedia. A altura e comprimento do tálus, assim como o grau de subluxação talonavicular, foram medidos utilizando-se um sistema de retas traçadas a partir de pontos anatômicos objetivos e comparados por meio de porcentagem simples sobre as medidas dos pés contralaterais. Este método foi idealizado de forma conjunta com um médico radiologista especializado no estudo do sistema musculoesquelético.

Os demais parâmetros, ou seja, as características da deformidade da cabeça e dômus do tálus e o padrão de seu trabeculado ósseo, são constatados por observação direta, em comparação com a anatomia normal do pé não afetado. No caso da deformidade da cabeça e dômus talar, os parâmetros podem ainda ser aferidos objetivamente, por meio de um instrumento tão simples como a régua de círculos concêntricos de Mose. Acreditamos que estes sejam parâmetros simples e objetivos, de fácil aplicação e, provavelmente, com bom índice de reprodutibilidade.

Um possível fator limitante de nosso trabalho talvez seja o pequeno número absoluto de pacientes incluídos, além da falta de correlação clínica dos resultados radiográficos, a qual pretendemos realizar em um estudo posterior.

Kalenderer et $a l^{(11)}$ constataram alterações significativas do comprimento e altura do tálus em todos os pacientes de sua série, assim como deformidades do dômus e da articulação talonavicular em sua grande maioria. Entretanto, não

\section{REFERÊNCIAS}

1. Dobbs MB, Gurnett CA. Update on clubfoot: etiology and treatment. Clin Orthop Relat Res. 2009;467(5):1146-53.

2. Mahmoodian R, Leasure J, Gadikota H, Capaldi F, Siegler S. Mechanical properties of human fetal talus. Clin Orthop Relat Res. 2009;467(5):1186-94.

3. Pearce DH, Mongiardi CN, Fornasier VL, Daniels TR. Avascular necrosis of the talus: a pictorial essay. Radiographics. 2005;25(2):399-410.

4. McKay DW. New concept of and approach to clubfoot treatment: section II correction of the clubfoot. J Pediatr Orthop. 1983;39(1):10-21.

5. McKay DW. New concept of and approach to clubfoot treatment: section I principles and morbid anatomy. J Pediatr Orthop. 1982;2(4):347-56.

6. Napiontek M. Clinical and radiographic appearance of congenital talipes equinovarus after successful nonoperative treatment. J Pediatr Orthop. 1996;16(1):67-72.

7. Farsetti P, De Maio F, Russolillo L, Ippolito E. CT study on the effect of different treatment protocols for clubfoot pathology. Clin Orthop Relat Res. houve correlação clinicorradiográfica significativa destas alterações, uma vez que todos os seus pacientes obtiveram resultados bons, sem limitações às atividades e com satisfação dos mesmos e de seus pais quanto à função e cosmética. Segev et $a l^{(12)}$ também encontraram alterações significativas no tamanho do tálus em pés tratados conservadoramente, sem comprometimento clínico equiparável.

Consideramos de grande importância a quantificação e o estabelecimento de parâmetros descritivos da deformidade do tálus, devido à sua implicação clínica. O achatamento do dômus talar altera a articulação tibiotársica com consequente diminuição da flexoextensão do pé. A deformidade e subluxação talonavicular está associada a uma diminuição da mobilidade do mediopé. A longo prazo, pode se estabelecer a osteoartrose, que pode evoluir com dor e limitação funcional.

Entretanto, não podemos afirmar que estas alterações sejam o resultado de uma necrose avascular, uma vez que podem representar simplesmente mais uma das características da doença. Um elemento que favorece esta teoria é o fato de que algumas destas deformidades ocorrem tanto em pés submetidos ao tratamento conservador quanto ao tratamento cirúrgico. Além disso, estas alterações não se enquadram nas características normalmente observadas na osteonecrose do tálus, representadas pelo aumento da densidade, fragmentação e colapso ${ }^{(9)}$.

\section{CONCLUSÃO}

As alterações talares foram observadas em 100\% dos pés tratados pela técnica de McKay nesta amostra. O estabelecimento de parâmetros radiográficos para descrever e quantificar as deformidades do tálus mostrou-se viável, por meio de técnicas simples e de fácil execução. Novos estudos são necessários para se determinar a correlação clinicorradiográfica destes achados, bem como avaliar sua reprodutibilidade e aplicabilidade em amostras maiores.

2009;467(5):1243-9.

8. Ponseti IV, El-Khoury GY, Ippolito E, Weinstein SL. A radiographic study of skeletal deformities in treated clubfeet. Clin Orthop Relat Res. 1981;(160):30-42.

9. Cummings RJ, Bashore CJ, Bookout CB, Elliott MJ. Avascular necrosis of the talus after McKay clubfoot release for idiopathic congenital clubfoot. J Pediatr Orthop. 2001;21(2):221-4.

10. Ippolito E, Fraracci L, Farsetti P, Di Mario M, Caterini R. The influence of treatment on the pathology of club foot. CT study at maturity. J Bone Joint Surg Br. 2004;86(4):574-80.

11. Kalenderer $\mathrm{O}$, Reisoglu A, Turgut A, Agus $\mathrm{H}$. Evaluation of clinical and radiographic outcomes of complete subtalar release in clubfoot treatment. J Am Podiatr Med Assoc. 2008;98(6):451-6.

12. Segev E, Yavor A, Ezra E, Hemo Y. Growth and development of tarsal and metatarsal bones in successfully treated congenital idiopathic clubfoot: early radiographic study. J Pediatr Orthop B. 2009;18(1):17-21. 